\title{
Téoros
}

Revue de recherche en tourisme

\section{Les systèmes informatisés de réservations dans l'industrie touristique}

\section{Stanley Tremblay}

Volume 9, numéro 3, novembre 1990

La distribution du produit touristique

URI : https://id.erudit.org/iderudit/1079885ar

DOI : https://doi.org/10.7202/1079885ar

Aller au sommaire du numéro

Éditeur(s)

Université du Québec à Montréal

ISSN

0712-8657 (imprimé)

1923-2705 (numérique)

Découvrir la revue

Citer cet article

Tremblay, S. (1990). Les systèmes informatisés de réservations dans l’industrie touristique. Téoros, 9(3), 14-18. https://doi.org/10.7202/1079885ar d'utilisation que vous pouvez consulter en ligne.

https://apropos.erudit.org/fr/usagers/politique-dutilisation/ 
Au cours de la deuxième moitié du XXe siècle, le monde du voyage et des communications a connu une évolution technologique exceptionnelle. Les mêmes facteurs socio-technologiques furent lescatalyseurs qui expliquent le remodelage des réseaux de distribution de l'offre touristique.

Les communications nous ont conduit non seulement à la convergence de l'information, actualisant ainsi le "itvillage global" projeté par McLuhan, mais aussi à la mondialisation des marchés grâce aux échanges de données électroniques permettant les transactions en temps réel, peu importent les distances géographiques. Ces technologies de l'information régularisent déjà une grande partie des échanges dans l'industrie touristique.

Nous pensons mểme que l'impact de l'informatique et des télécommunications sur les techniques de la distribution touristique modifiera de façon notable le domaine des stratégies commerciales traditionnelles des prestataires et des fournisseurs. De fait, les réseaux actuels de la distribution des produits touristiques sont déjà irrémédiablement changés. Même si certains faits échappent encore à notre observation, le phénomène n'en demeure pas moins réel. Et l'ignorance de ces réalités risque d'être lourde de conséquences.

Un rapport fort révélateur, remis à Tourisme Canada en 1988, affirmaitqu" "un regard rapide montre que $l^{\prime}$ 'industrie canadienne du tourisme semble accuser un retard vis-avis de ses concurrents, en particulier en matière de fourniture d'information, de capacité d'analyse des marchés, et d'interconnexion entre systèmes et bases de données (...). Par conséquent, des lacunes dans ces domaines seront très apparentes et surtout auront une incidence sur la position concurrentielle perçue et réelle"(i). Cette absence relative de connaissance des nouveaux systèmes d'informations et de réservations en tourisme risque d'être coûteuse pour les fournisseurs au cours des prochaines années. Nous nous proposons d'explorer ces systèmes afin d'en circonscrire les champs d'action actuels, tout en décrivant sommairement leur fonctionnement. L'éclairage sera de toute évidence modeste mais nous espérons qu'il aura le mérite de réduire la surface des zones ombragées.

\section{Le SIR mal connu}

Un monde qui se dérobe à notre regard est celui des systèmes informatisés de réservations (SIR). Issus des architectures technologiques des grands transporteurs aériens des années ' 60 , ils s'êtalent maintenant sur la surface du globe, pénétrant progressivement tous les points de distribution des marchés touristiques. Aucun passager de vols réguliers $n^{\prime} a$ totalement échappé à leur emprise au cours de la dernière annce. Bientôt les touristes qui y échappent feront sans doute l'objet d'un nouveau segment de marché: celui des insoumis. Certains auteurs affublent ces systèmes du nom de "Big Brother" en référence bien sûr au célèbre ouvrage de $\mathrm{G}$. Orwell ${ }^{(2)}$. Quoi qu'il en soit, le traitement informatique des données en tourisme ne va pas disparaître sous le poids des étiquettes. Au contraire, la tendance révèle davantage une progression dans la diversification des fonctions. Ainsi, une typologie des systèmes commence à s'imposer. Selon leurs configurations technologiques et leur mission respective, nous identifions 3 types de systèmes: les systèmes de distribution globale (GDS); les systèmes de réservations des établissements hôteliers; les systèmes de réservations de destination touristique. Force est de reconnaittre que les frontières démarquant la spécificité propre de ces systèmes deviennent difficiles à identifier avec les développements accélérés de chàcun tant du point de vue technologique que du point de vue commercial. Et puis ils ont pris pour habitude de "communiquer", grâce aux technologies d'interconnexion, ce qui ne prédispose guère à la transparence des frontières.

\section{Les grands et les très grands SIR}

Nous sommes redevables à la compagnie aérienne American Airline d'avoir introduit le premier système informatisé de réservations en 1964. De nombreux autres transporteurs allaient, dans les années qui suivent, développer des systemes similaires

"Monsieur Stanley Tremblay est président d"Alpha 90. 

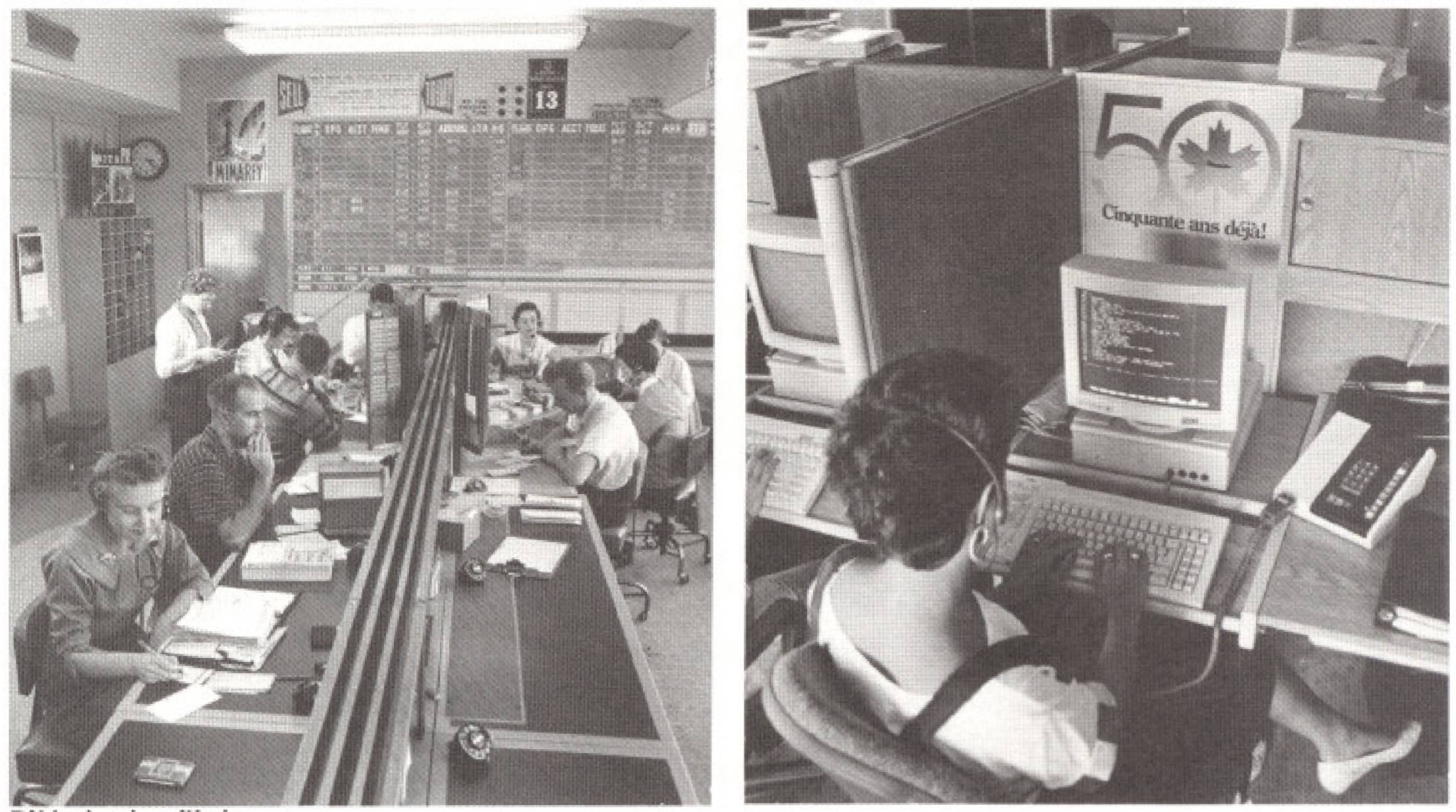

D'hier à aujourd'hui ...

(voir le tableau 1). À l'origine, les applications utilisaient la même base mise en marché par IBM, et connue sous le nom de PARS: Passenger Airline Reservation System (à ne pas confondre avec le produit PARS de TWA). Labase demeure la même aujourd'hui, en ce sens que les systèmes sont configurés pour gérer des transactions multiples simultanément avec de nombreuses modifications effectuées fréquemment à la base centrale de données.

Cettepremièrephase leur permit de déployer la technologie chez les distributeurs naturels que représentaient les agents de voyage. Puis vers le milieu des années '70, les enjeux de la distribution prennent une tournure dramatique pour la réservation des produits du voyage avec l' introduction de l'ultraswitch qui est un système d'interface permettant de relier entre eux les ordinateurs de réservations aériennes aux ordinateurs de toute autre banque de données de produits touristiques. On pouvait désormais réserver son billet d'avion, sa chambre d'hôtel et une voiture chez l'agentde voyages qui accédait directement avec son clavier-écran à des bases de données pour effectuer les transactions. Doiton rappeler que les caractéristiques de ces systèmes permettent la recherche multicritères, ce qui garantit aux clients une recherche exhaustive et instantanée des services. Selon les critères de choix des plus capricieux! Avec en prime des confirmations de ses réservations en temps réel: on met à peine quelques secondes à réaliser les transactions. La parfaite connexion était née; $\mathrm{l}$ 'intermédiation devenait presque complète. À partir de ce moment, les stratégies commerciales s'orientent sur la pénétration du plus grand nombred'agences possible avec le plus grand nombre de claviers-écrans possible. Nés pour faciliter la tâche des agents de voyage, aujourd' hui ces systèmes rayonnent dans l'industrie touristique et imposent, dit-on, partout des choix qui prennent la forme de véritables cassetêtes chinois pour les agents.

Le plus récent bilan nous révèle que quatre très grands systèmes s'imposent dans un univers qui n'a cure de former des alliances: APOLLO, SABRE, SYSTEM I et WORLDSPAN ${ }^{(3)}$. Leur impact dans le domaine du voyage est majeuretce, d'autant plus qu'ils projettent tous d'introduire la distribution directe aux consommateurs. À titre indicatif, soulignons qu' aux États-Unis les SIR transigeaient $82 \%$ des réservations aériennes, $77 \%$ des sièges de vols internationaux, $68 \%$ des locations de voitures et $51 \%$ des réservations des chambres ${ }^{(4)}$. Se demande-t-on encore s'ils existent vraiment!

Mais illustrons de manière encore plus concrète la puissance de ces systèmes et de leur impact en décrivant la feuille de route de SABRE: le système peut traiter 2000 messages/seconde, ileffectue 500000 réservations par jour et conserve en banque 45 millions de tarifs; il maintient a jour une banque de données incluant 650 compagnies aériennes, 21000 hôtels, 52 compagnies de location de voitures et donne accès à 8 sociétés de chemin de fer. Ouf! Nous sommes presque tentés de passer sous silence le fait qu'il permet de réserver des billets de théâtre, des billets pour des événements, la location de limousines et quoi d'autre encore... depuis hier. Son réseau de distribution auprès des agents de voyages est tout aussi imposant avec 14500 terminaux dans 47 pays. Double ouf! Les deux autres GDS (Global Distribution Systems) possèdent des caractéristiques comparables ou potentielles. Ces mega-systèmes ne sont pas sans inquiéter les observateurs les plus perspicaces, mais leur efficience ne justifie sans doute pas à elle seule les anxiétés exprimées. Rappelonsqu'ils ne sont pas les seuls dans le monde de la distribution et de l'intermédiation électronique.

\section{Les SIR de I'hébergement}

La grande hôtellerie, comme la moyenne d'ailleurs, a aussi mis en place des systèmes de réservations. Ceux-ci nous semblent plus familiers, non sans raison, puisqu'ils nous sont directement accessibles comme consommateurs. Quidenous n'a pas utilisé une ligne 1 - 800 pour réserver une chambre? Mais derrière cette transaction qui est transparente, des opérations d'échanges de 


\section{TABLEAU 1}

Les GDS

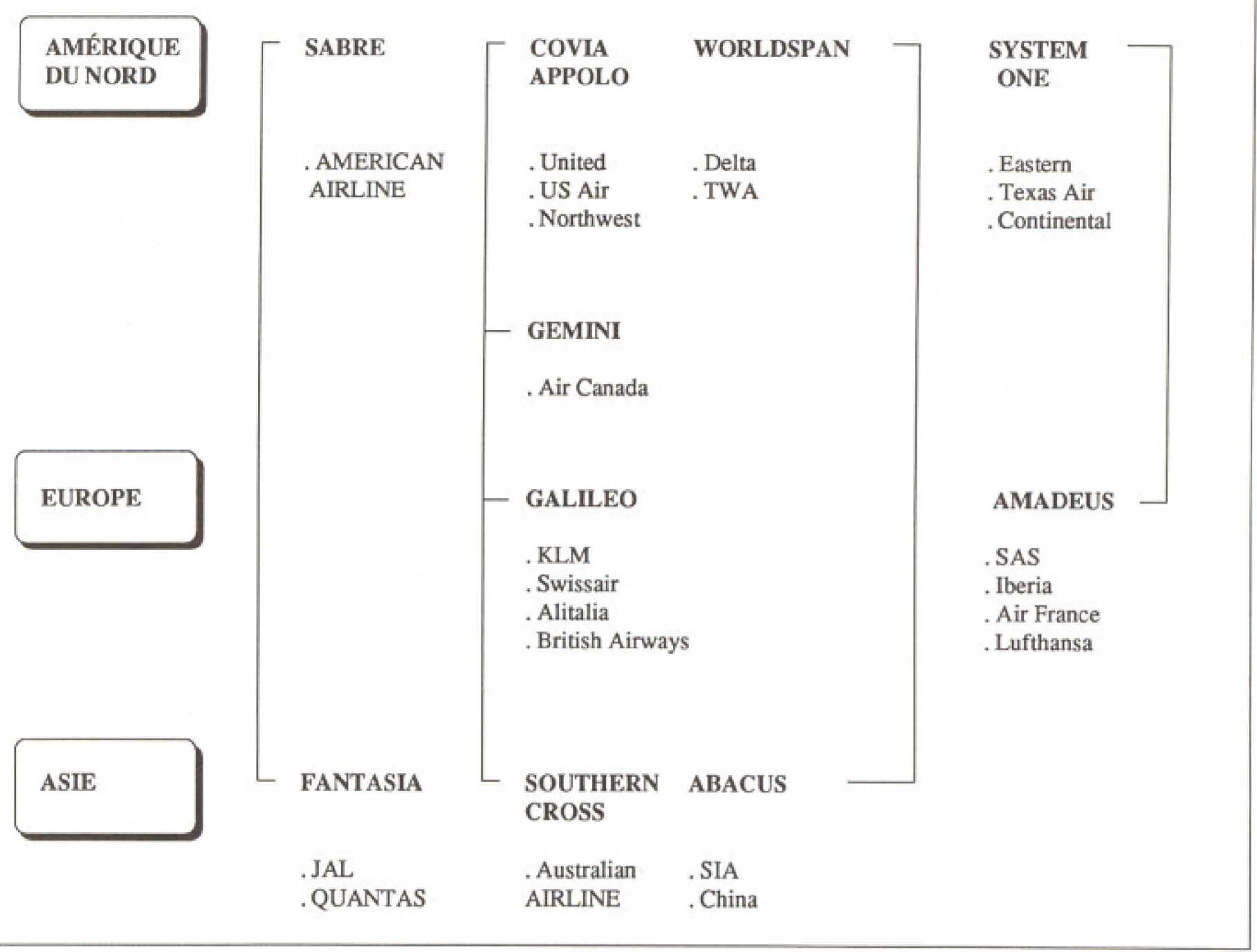

données parfois très complexes supportent notre démarche.

Nous pensons que le meilleur est à venir dans le secteur hôtelier. Un vent de renouveau technologique souffle dans ce secteur. Ce renouveau nous incite à croire qu' ${ }^{\prime}$ ne véritable révolution est à se fomenter.

Si l'explosion des voyages-personnes depuis trente ans a stimule la mise en oeuvre de GDS, il fallait bien loger tous ces touristes. La croissance des immobilisations dans le domaine hótelier fût imposante au cours de cette même période. En 1988, le nombre de chambres au niveau mondial était estimé à 10,1 millions ${ }^{(5)}$. Le regroupement sous différentes bannières connut une relance au cours de la dernière décen- nie tout comme la tendance à la spécialisation auprès de segments de clientèles.

Pour les établissements d'hébergement, il était impératif de raffiner la distribution afin de "fidéliser" les clientèles et de protéger les parts de marché. Des systèmes de réservations se devaient d'être mis en place pour garantir aux clients un service rapide et fiable en fonction des disponibilités. Yrai aussi que des bases de données devaient être constituées pour alimenter les stratégies de marketing. La suite coule de source.

Toute chose égale par ailleurs, ces systèmes tardent à être aussi performants. Avouons que la gestion des disponibilités d'un hôtel présentent des défis que n'ont pas à confronter les transporteurs. Et puis, rares étaient les corporations - dont le capital est moins centralisé que les compagnies aériennes - susceptibles d'investir les centaines de millions de dollars nécessaires au développement.

Du strict point de vue technologique, les systèmes évoluent selon des principes tout à fait differents: l'inventaire des chambres n'est jamais (disons presque jamais pour éviter les critiques) modifié directement par le système central. La gestion de l'inventaire demeure la prérogative incontestée de la direction de l'établissement contrairement au SIR des transporteurs ${ }^{(6)}$. Le processus décisionnel implique un certain arbitraire de la part du gestionnaire. Cet arbitraire se traduit par l'utilisation de 
la technique du "sell/no sell" selon laquelle on ouvre ou ferme l'inventaire à $n$ 'importe quel moment. Ceci s'explique en partie par la pratique du "yield management". Cette technique de gestion a pour prémices que chaque chambre doit être louée au meilleur tarif à tout moment. A la limite, la politique de tarifs des chambres est influencée par les conditions locales du marché sur une base quotidienne.

$\mathrm{N}^{\prime}$ avançons pas pour autant que ces systèmes incorporent des carences qui les rendent inefficaces. II y a de nombreuses centrales de réservations hôtelières de grande envergure. Afin de camper leur importance dans le domaine de la distribution, nous nous limitons à la description des mieux connus.

La chaine Best Western International offre un service de réservations à plus de 3400 établissements indépendants. La banque de données conserve et modifie quotidiennement les tarifs de plus de 260 000 chambres. Il s'effectue au-delà de 9 millions de réservations annuellement $(7)$. Pour sa part, UTELL INTERNATIONAL est dédiée exclusivement aux agents de voyages qui communiquent avec une trentaine de bureaux de réservations à travers le monde. La banque de données comportent les profils de plus de 8000 établissements hôteliers à travers le monde ${ }^{(\theta)}$. Un système très sophistiqué permet de confirmer plus d'un million de réservations annuellement. Pour l'agent de voyages, la confirmation s'obtient en quelques minutes, le préposé exécutant la transaction avec un clavierecran muni d'un appareil vidéodisque projetant les images de l'établissement et desdonnées afférentes. L'espace manquant, nous nous devons de passer sous silence de très grands systemes tels HOLIDEX 2000 (Holiday Inn), RESERATRON II (Sheraton), MARSHA (Marriott).

Beaucoup de grands systèmes hóteliers subissent présentement des refontes importantes. Le renouveau, dont nous faisions mention plus haut, s'oriente vers une technologie de pointe, vers des alliances qui font de ces systèmes de véritables partenaires des GDS et les plus avantgardistes commencent a explorer la distribution directe aux consommateurs. Nous sommes tentés d'affirmer que l'éventuelle intégration des principes du "yield management" dans les fonctionnalités des systèmes de centrales hôtelières représentera une évolution réelle dans le monde des SIR. Le temps se fait pressant pour les hôteliers ${ }^{(9)}$.

\section{Les nouveaux arrivants: les SIR- destination}

De nombreuses destinations touristiques étudient présentement l'opportunité de mettre en place des systèmes informatisés de renseignements et de réservations pour les touristes. Comme nous l'avons vu, la technologie des SIR est éprouvée. En un sens, les "nouveaux arrivants" bénéficient de nombreux ayantages pour le développement des architectures technologiques. Deconception plus récente, soit đu début des années ' 80 , ils évolueront sans doute rapidement en raison des progrès remarquables qu'a connu la microinformatique ces trois dernières années. S'offrent aussi aux gestionnaires du développement de ces systèmes des opportunités d'accéder directement aux technologies de pointe en achetant tout simplement les applications existantes. Mais ne perdons pas de vue que la majorité de ces systèmes sont présentement en gestation. Les plus anciens n'ont guère plus de dix ans d'expérience. Cet état de fait nous oblige à une certaine prudence lorsque vient le temps de prévoir leur évolution à plus long terme. Toutefois, les objectifs de ces systèmes nous permettent d'anticiper leur trajectoire pour les prochaines années.

Les SIR de destination touristique s'expliquent par le souci non seulement de mettre en marchế la totalité de l'offre touristiqued'une destination, mais ausside favoriser un accueil de premier ordre fondé sur un service de qualité. $\mathrm{N}^{\text {'hésitons pas à }}$ l'affirmer, le fondement stratégique de tels systèmes estdouble: offensifet défensif. II se veut offensif par l'occasion qu' ils offrent dans le positionnement de la destination; il est deffensif, puisqu'ils cherchent à limiter la domination de systèmes de distribution sur lesquels les fournisseurs n'ont aucun contrôle. À cet égard, peut-être apportentils une réponse dynamique aux craintes de nombreux fournisseurs d'être les grands oubliés des nouveaux réseaux de distribution. De fait, l'offre touris tique se compose de multiples petits établissements répartis géographiquement sur de grands espaces, ne rendant pas leur intégration attrayante pour les GDS. Les coûts de gestion deviennent énormes.

Les SIR-destination ont une configuration $s$ 'inspirant de leurs "grands frères" tout en possédant une personnalité propre. À certains égards, ils se comportent comme des centrales hôtelières mais en regroupant l'ensemble des établissements d'hébergement par des réseaux de microserveurs. Par ailleurs, ils agissent comme des GDS en ce qui a trait aux réservations de tout type de prestations touristiques mais sur un territoire délimité. Du point de vue fonctionnel, ils effectuent des réservations, ils exécutent de l'intermédiation et ils dispensent des renseignements et des informations touristiques. Une dernière particularité: jusqu'à maintenant, ils furent, tous sans exception, réalisés grâce à la collaboration des gouvernements avec des entreprises privées. Cette particularité s'explique par la responsabilité assumée par les gouvernements dans lapromotion et dans la gestion des fonctions d'accueil de la majorité des destinations touristiques.

Quel touriste canadien ne connait pas le service CHECK INNS de la NouvelleÉcosse ${ }^{100}$ ? Renommé pour la qualité de l'accueil et des renseignements, il est aussi un bon système informatisé de réservations. Il possède les principales fonctionnalités des applications de première génération ce qui lui permet d'assumer des transactions de réservations de chambres. On accède au service par lignes téléphoniques.

Pour sa part, le système australien ATLAS (Queensland Tourist \& Travel Corporation, une société gouvernementale) possède une configuration technologique qui le rapproche des systèmes de pointe(11). Fonctionnant sur un réseau de microordinateurs, il est en interconnexion avec les SIR des distributeurs et des fournisseurs lui permettant ainsi d'effectuer des réservations pour l'ensemble des prestations touristiques. De plus, il offre une multitude de fonctions de gestion qui représentent une valeur ajoutée importante. Plus près de nous, le ministère du Tourisme du Québec et des partenaires du secteur privé(12) complètent présentement une étude de faisabilité visant la mise sur pied d'un système informatisé de réservations. Le projet actuel $s$ 'orientera vers une configuration technologiquequi intègre les fonctionnalités que possèdent les systèmes les plus performants: renseignements, interconnexion, intermédiation, réservations, paiement, gestion des données. Cen'estd'ailleurs pas le seul projet a l'étude présentement au Canada. Bref, les jeux sont faits!

\section{La prochaine conquête des SIR}

La distribution informatisée en tourisme s'appuie sur des infrastructures puissantes qui garantissent son existence et son expansion. Nous devrions même anticiper de plus profonds changements dans les méthodes de distribution compte tenu du déploiement irréversible des réseaux télématiques. Déjà, des fournisseurs et grossistes s'adressent directement aux 
consommateurs (Nouvelles Frontières et Club Med en France entre autres). Le réseau Minitel distribué largement dans les foyers français ne compte pas moins de 350 services d'informations et de renseignements; le 3615 est utilisé par 30 serveurs de réservations touristiques ${ }^{(13)}$. Si l'expansionestmoins évidente en Amérique du Nord, le réputé SABRE s'introduit dans le secteur de la distribution directe avec son produit EAASY SABRE accessible présentement sur des serveurs tels Prodigy, Genie, CompuServe et Dialcom ${ }^{(1)}$. On notera qu'il est question ici d'usagers utilisant leur micro-ordinateur professionnel ou personnel pour transiger avec des serveurs à multiples services. Dans ce secteur aussi, demain, c'est maintenant!

A la lumière des progrès accomplis dans le domaine de la distribution informatique en tourisme et des développements en cours dans le secteur des technologies axées sur le consommateur (telles la télévision interactive et la carte à mémoire), nous anticipons des changements significatifs au niveau des stratégies en marketing touristique. Les prochaines années (cinq tout au plus) seront déterminantes pour chacun des intervenants qui devront maintenir des contacts avec les réseaux traditionnels tout en préparant une rupture stratégique. Mais vers quoi? Quels changements sont prévisibles?

\section{Les SIR exigent des changements}

Les stratégies de marketing sont déterminantes en tourisme. Nous ne sommes pas dans le domaine des achats impulsifs. Ces stratégies s'expriment donc par des agencements, les uns plus subtils que les autres, de programmes et d'activités de mise en marché. Afin de mesurer l'impact probablesur les méthodes actuelles, passons en revue les principaux éléments composant. les programmes de marketing touristique habituels, en proposant les orientations que ceux-ci sont susceptibles de prendre.

La publicité institutionnelle s'orientera encore davantage sur des axes qui précisent l'identité de la destination ou de l'équipement, tout en recherchant à créer "du vécuémotif", en s'adressant aux cordes sensibles du public cible ${ }^{(15)}$. Les renseignements, les informations et les points de distribution des produits feront simplement l'objet d' une référence d'accès permettant au consommateur de terminer sa recherche et de faire la trans action. Ence qui a trait aux forfaits, nous croyons que les grossistes réceptifs, comme les toursopérateurs (voyagistes), répondront progressivement à la demande des consommateurs désirant préparer leur plan de voyage eux-mêmes, en évoluant vers le "sur mesure". La distribution électronique se prête très bien à cette technique déjà en usage pour des groupes de clients particuliers.

L'information prendra de nouvelles configurations. Tant au niveau du contenu qu'au niveau de la forme, les renseignements subiront une transformation pour s' adapter au mode de diffusion électronique. Le videotex, le video-disque ou les écransmultiples commanderont aux fournisseurs de rechercher une rédaction qui a peu en commun avec le support papier actuel (SABRE a déjà mis en marché le produit Official Recreation Guide qui n'est rien de moins qu'un guide touristique international ${ }^{(16)}$ ). Foumisseurs et grossistes verront à décrire les produits de manière à créer une adéquation presque parfaite entre les bénéfices recherchés par $1^{3}$ acheteur potentiel et les caractéristiques de l'offre. Défi de taille compte tenu du médium électronique.

Ces mêmes fournisseurs feront la gestion de leurs réservations avec des microordinateurs. La planification des budgets annuels, cauchemars des directeurs des ventes de l'hôtellerie, déterminera les projections en incorporant les ventes par réseaux d'interconnexion avec les SIR, incluant les réseaux de clients corporatifs, et en utilisant des banques de données internes qui contiennent les profils et les habitudes des clients. Tout en utilisant les principes du "yield management" pour optimiser les revenus de l'établissement! Ces projections feront le bonheur des directeurs généraux comme de propriétaires et les directeurs des ventes pourront enfin dormir!

En conclusion, le monde des SIR n'est pas une illusion. Certains garderont leur montre irrémédiablement à l'heure de 1980 , en reformulant les mêmes postulats stratégiques en 150 pages au lieu des 100 pages d'alors; d'autres, stimulés par ces nouvelles technologies, voudront faire appel à la "magie" des ordinateurs pour tout solutionner rapidement. Les erreurs stratégiques des entreprises, tant gouvernementales que privées, deviendront coûteuses si on refuse d'initier les changements qui s'imposent. Il faut $\mathrm{s}$ 'inspirer des méthodes de gestion proactives etgarder une vision juste des choses par un état de vigie constant. Les transformations souhaitables exigeront des investissements en temps, en savoir-faire et en capital. La rentabilité future de l'exploitation de nombreux établissements touristiques en dépend. Les enjeux sont clairs.
Notes explicatives

(1) James F. HICKLING, Managements Consultant Ltd. Applleatlons de la technologie dans rindustrie du tourisme. Fapport principal, próparé pour Tourisme Canada, Ottawa, juin 19e8, Le rapport représente un excellent inventaire des technologies applicables a l'industrie. Un bilan sommaire permet de juger du retard du Canada

(2) Josquin BARRE, Big Brother ou to plualisme, Tourismatique, Paris, juinjuillet 1990.

13) Brunot TAEVIDIC, La planëte systeme, L'Écho touristique, Paris, 22 juin 1990. Note: Dans le mème ordre d'idee, il est certes intéressant de signaler la récente alliance (dans l'esprit de libreéchange nul doute), de GEMINI, le seul SIR canadien, avec COVIA-APOLLO des Etats-Unis. Le système RESERVEC, développé à l'origine par Air Canada, distribuait uniquement aupres des agences de voyages canadiennes. Le SIR canadien joint donc les rangs des GDS. Rebaptisé "APOLLO BY GEMINI" depuis le mois de juin 1990 , le nouveau systeme fait liobjet de modifications importantes: au niveau des fonctionnalites. Une petite touche rafraichissante pour les agents de voyages francophones du Québec: les références pour les usagers et les reglements sur les tarifs sont presentés dans des tableaux bilingues.

(4) Pauline J. SHELDON, Les réseaur de distribution des voyages aux États-Unis, Téoros, wol. 9, no 2, juillet 1990

(5) James MCGUFFIE, CAS Developmentand the Hotel Sector, Travel \& Tourism Analyst, Londres, juin 1990.

(6) David WARDELL, Hotel Technology and Reservation Systems, Travel \& Tourism Analyst. Londres, juin 1987.

(7) James McGUFFIE, op. cit.

(8) Jacques RIPEAU, UTELL INTERNATIONAL Rapport de visite pour le projet CRTQ Montreal, mars 1990.

(9) David WARDELL, op. cit.

(10) CHECK INNS (nom de la sociétel ou CHECK IN, a des contrats de service avec $98,9 \%$ des établissements höteliers de la Nouvelle-Ecosse. La Direction du marketing du ministère du Tourisme de la $N$. E nous informait recemment que le service couvre desormais l'ensemble des provinces des Maritimes.

(11) Peter SMITH Atlas Presentation, Australian Trave \& Leisure Automated Systems, Sydney, juin 1990

(12) Les partenaires du ministère du Tourisme du Quebec sont la Conféderation des caisses populaires et d'économie Desjardins du Quebec qui assure la gestion de l'étude de faisabilite, le groupe D.M.R., les ordinateurs Tandem Canada, le ministere des Cormmunications du Québec et les. associations touristiques regionales.

(13) Tourisme et Communications, Paris, janvier 1990

(14) Cathryn CONAOY, A Tour of the Big Three, Online Access, vol. 5, no 2, Chicago, automne 1990.

(15) Jay Conrad LEVINSON, Guerlila Marketing. Paris, First, 1969. L'ouvrage est d'abord paru chez Houghton Miffin Co, la méme annede.

(16) SABRE, Official Recreation Guide, 1990 